\title{
Alkyltransferase and Aryltransferase
}

National Cancer Institute

\section{Source}

National Cancer Institute. Alkyltransferase and Aryltransferase. NCI Thesaurus. Code C20075.

Alkyltransferases and Aryltransferases are classes of enzymes that transfer an alkyl or aryl chemical group from one compound (the donor) to another compound (the acceptor). Alkyls are radicals derived from an aliphatic hydrocarbon by removal of a hydrogen atom. Aryl are radicals derived from an aromatic compound by removal of a hydrogen atom. 